\title{
Cabotaje interamericano. Una propuesta de solidaridad continental en tiempos de guerra elaborada por el Museo Social Argentino $(1914-1916)^{1}$
}

Guillermina Zanzottera ${ }^{2}$

\section{Resumen}

El artículo analiza las estrategias desplegadas por el Museo Social Argentino ante el impacto de la Primera Guerra Mundial en América Latina. Esta institución privada, orientada al bien público, asumió como propia la responsabilidad de promover una campaña de solidaridad continental, proponiendo la necesidad del aislamiento pacifico de América y la neutralización del tráfico marítimo interamericano. Frente al conflicto bélico el Museo Social Argentino hizo un llamamiento a los Estados, a la intelectualidad y a las instituciones sociales americanas para que inicien una acción conjunta, iluminado una época de límites difusos donde la acción oficial se solapaba con las iniciativas privada.

Palabras clave: Museo Social Argentino Cabotaje interamericano - Solidaridad Continental - Neutralidad Americana - Primera Guerra Mundial

\begin{abstract}
This article analyses the strategies unfolded by Museo Social Argentino at the impact of the First World War in Latin America. This private institution, oriented to the public well-being, took the responsibility of promoting a continental solidarity campaign, proposing the need for a Pacific isolation of the Americas and the neutralization of Inter-American marine traffic. In the face of the armed conflict, Museo Social Argentino made an appeal to the different States, intelligentsia and american social institutions to initiate a joint action, enlighten on a period of dim limits where official action overlapped those of private initiatives.
\end{abstract}

Keywords: Museo Social Argentino Inter-American cabotage - Continental Solidarity - American Neutrality - First World War

\footnotetext{
${ }^{1}$ Trabajo recibido el 30/07/2016. Aceptado el 02/11/2016.

${ }^{2}$ Licenciada en Ciencia Política (Universidad de Buenos Aires) y Magíster en Ciencias Sociales (Universidad Nacional de Luján). Contacto: guillezanzo@gmail.com
} 


\section{Introducción}

El Museo Social Argentino ${ }^{3}$ fue una institución privada orientada al bien público que en los comienzos de la Primera Guerra Mundial promovió una campaña de solidaridad continental en favor de los derechos y garantías de los países neutrales. A partir de esas acciones se vinculó con cónsules, diplomáticos, instituciones sociales, empresarios e intelectuales latinoamericanos, lo que le permitió constituir una robusta red de personas, saberes y problemas que fueron configurando los rasgos de un núcleo de preocupaciones y que posibilitaron la construcción de la noción de solidaridad que fue clave en las ideas que intentó impulsar. De esta manera, el MSA, que se constituyó como una red de redes, una institución federadora que anudaba personas y problemas, operó como una caja de resonancia de los debates nacionales e internacionales. En ese sentido, la propuesta que se despliega entre los años 1914 y 1916 se inserta, por un lado, en un momento de amplias movilizaciones sociales vinculadas a la guerra ${ }^{4} \mathrm{y}$, por el otro, en un contexto de neutralidad latinoamericana, ${ }^{5}$ donde la preocupación por las consecuencias económicas de la guerra lleva a la dirección del MSA a interrogarse por el rol que deberían cumplir las instituciones sociales latinoamericana en el escenario de la guerra europea.

Para llevar adelante este estudio nos centramos en el análisis de los libros Museo Social de Buenos Aires. Fundamentos y Anteproyecto, escrito por Tomas Amadeo, publicado en 1910, y El Aislamiento Pacifico de América, publicado en $1916^{6}$ por el MSA, y de los Boletines del Museo Social Argentino entre los años 1912 y $1916 .^{7}$

\footnotetext{
${ }^{3}$ En adelante MSA. Sobre el MSA ver: Girbal de Blacha y Ospital,(1986); Novick,(1993) y Pelosi,(2000).

${ }^{4}$ Esta primera etapa es analizada por Tato,(2010) como de «asociacionismo solidario».

${ }^{5}$ El vacío existente sobre las fluidas interacciones que la Primera Guerra Mundial generó entre los países Latinoamericanos está siendo subsanado con la aparición de nuevas líneas de investigación enmarcas en los estudios promovidos por la conmemoración de los cien años del inicio del conflicto bélico, ver: Riken,(2014); Tato(2014); Compagnon,(2014); Ramírez Bacca,(2015) y Ojeda Revah,(2014).

${ }^{6}$ Recoge la documentación relativa a la campaña a favor de la neutralización del tráfico marítimo interamericano.

${ }^{7}$ El Boletín era el órgano de difusión del MSA, el análisis de su contenido nos permite observar el desenvolvimiento de las ideas y estrategias de la institución. A fines de 1914 en el Boletín inaugura una sección llamada El Museo Social Argentino y la neutralidad Americana (cabotaje interamericano) que tiene una aparición regular hasta fines de 1915 y durante 1916 aparece una nueva sección llamada Cabotaje interamericano (el aislamiento pacifico
} 
De esta manera, en primer lugar examinaremos sucintamente las características del MSA, para luego detenernos en el impacto que implicó la guerra en las acciones que la institución estaba desarrollando, lo que nos permitirá analizar la propuesta de solidaridad continental y esbozar unas conclusiones.

\section{El Museo Social Argentino}

El MSA fue fundado en el año 1911 a partir de un proyecto elaborado por el Ingeniero Agrónomo Tomas Amadeo. ${ }^{8}$ Operó como un espacio de sociabilidad en el que convergieron profesionales y especialistas, pertenecientes a diferentes organizaciones sociales, culturales y económicas, que aspiraron a articular un discurso reformista ${ }^{9}$ sobre los problemas sociales que atravesaban al país, lo que les permitiría presentarse como mediadores entre el Estado y la sociedad.

Si bien el MSA convocó a un gran número de personas e instituciones, ${ }^{10}$ sus rumbos estuvieron a cargo de un Consejo Superior, ${ }^{11}$ compuesto

de América), estas secciones fueron acompañadas por sendos artículos sobre el problema de la Guerra en Argentina y en Latinoamérica, que se extendieron hasta 1918. Por su parte en la sección Crónica y Archivo, se puede observar el posicionamiento de los miembros de la institución respecto a las vinculaciones Latinoamericanas.

${ }^{8}$ Amadeo (1880-1950) provenía de una familia terrateniente de la provincia de Buenos Aires en 1904 se recibió de Ingeniero agrónomo en la Facultad de Agronomía de la Universidad de La Plata y en 1921 se doctoró en jurisprudencia en la Facultad de Derecho y Ciencias Sociales de la Universidad de Buenos Aires (en adelante UBA). Fue un referente de la profesionalización agrícola en Argentina, se desempeñó como funcionario del Ministerio de Agricultura de la Nación, fue docente universitario y uno de los impulsores de la reorganización de la Facultad de Agronomía de la Universidad de La Plata luego del proceso de autonomización. Ver: Amadeo,(1939) y Pelosi,(2000).

${ }^{9}$ Las reformas sociales formaban parte de una red muy heterogénea que encontró un terreno común en las búsquedas de respuestas prácticas a los problemas sociales y que apuntaban a establecer nuevas relaciones entre el Estado y la sociedad y entre el trabajo y el capital, ver Topalov,(1990); Rodger,(1998) y Horne,(1998). El reformismo fue estudiado en Argentina como reformismo liberal - Zimmermann,(1995) - o conservador Suriano, (2000)- Estas interpretaciones contrapuestas, fueron convergiendo en los últimos años al calor de los estudios internacionales sobre el movimiento reformista de los inicios del siglo, ver Suriano,(2013) y Zimmermann,(2013).

${ }^{10}$ Para su fundación el MSA contaba con 650 miembros, de los cuales 255 eran activos y 395 adherentes, este número se mantendrá con pequeñas alteraciones hasta 1920.

${ }^{11}$ En adelante CS. 
por 15 consejeros -número que irá variando en las sucesivas modificaciones de estatuto- nombrados en Asamblea por los socios activos. En los años que nos ocupan, la Presidencia estuvo a cargo del Dr. Emilio Fres, como primer vicepresidente fue designado el Dr. Rodolfo Rivarola, quien a los pocos meses renuncia y en su lugar es elegido el Dr. Agustín Álvarez, quien ejerció el cargo hasta su fallecimiento en 1914, motivo por el que es designado, en noviembre de ese año, el Dr. Eleodoro Lobos, en tanto que Tomas Amadeo fue nombrado Secretario General.

La institución fue presentada como un Instituto de Altos Estudios Sociales orientado a la economía social, que tenía como objetivo principal recoger y trasmitir información sobre la cuestión social y promulgar el asociativismo como respuesta a los nuevos problemas. La preocupación de los socios fundadores se centraba en la carencia a nivel nacional de un organismo que centralice estudios e informes sobre los problemas sociales. Por lo que, intentando posicionarse como detentadores de un saber científico, se propusieron el estudio práctico de los problemas sociales y la organización de una biblioteca y archivo. La información recabada era, por un lado, clasificada, ordenada y puesta a disposición de los estudiosos y/o legisladores y, por el otro, sintetizada y puesta al servicio del pueblo. Esa última acción era considerada fundamental para que las obras sociales no fracasen ya que estimaban que era la masa del pueblo quien debía poder aprovechar los estudios y las iniciativas.

Además de esas tareas comunes a otros centros de estudio, el MSA, tenía la función particular de llevar adelante la propaganda argentina en el extranjero. Sus fundadores evaluaban que el país era mal conocido, por lo que se proponían organizar una propaganda centralizada y uniforme, basada en datos fidedignos, orientada de forma particular a Europa, Estados Unidos y América Latina, que subsane las falencias de propaganda oficial. $^{12}$

La fundación del MSA se daba en un momento de incipiente profesionalización del Servicio Exterior de la Nación, ${ }^{13}$ proceso en el estaban

\footnotetext{
${ }^{12}$ En el proyecto fundacional del MSA se reproducía una nota del diario La Nación de 1905 que recogía las opiniones de Emilio Frers sobre este tema: «Qué propagada ni qué informes, ni qué trabajo serios de ninguna clase, si muchos de nuestros cónsules o no conocen al país que representa y solo aspiran al nombramiento por razón de titulejo y de las ventajas que comporta, o no pueden hacer cosa alguna por defecto de instrucción, medios y recursos». Amadeo,(1910):58.

${ }^{13}$ Desde 1860 intenta reglamentarse el funcionamiento de Servicio Exterior de la Nación, ya que el del cuerpo diplomático no poseía una legislación que lo rigiera, ni que establecie-
} 
involucrados algunos de los miembros del Consejo Superior. Entre los que podemos mencionar a José León Suarez, ${ }^{14}$ director de la Revista Argentina de Derecho Internacional y fundador del Círculo de Estudios Diplomáticos y Consulares, iniciador de la cátedra de Derecho Diplomático y el creador, en $1907,{ }^{15}$ de la primera Escuela de Diplomacia del país, que funcionó en el ámbito de la Facultad de Derecho y Ciencia Sociales de la UBA. De esa carrera era profesor otro miembro del C.S del MSA el Dr. Ruiz Guiñazú. ${ }^{16}$ Por su parte, el Dr. Emilio Frers y Tomas Amadeo participaban en los debates sobre la calificación de los representantes designados en las oficinas de propaganda argentina en Europa.

Los miembros del MSA consideraban que la institución venía a cumplir una función trascendente, la de llenar «un verdadero vacío nacional», en lo que respectaba a la necesidad de un instituto «informador consultivo e intermediario de los problemas sociales». En definitiva, se proponían «colaborar con todas sus fuerzas a la obra del progreso nacional. Junto con los trabajos de los políticos y del parlamento y a la par de los cerebros de los pensadores que se afanan por verter sus ideas en el troquel común». ${ }^{17}$ Estas citas iluminan la concepción que sobre lo público tenían los miembros del Museo, lo que nos advierte sobre una época en donde instituciones privadas actuaban junto al Estado para solucionar los problemas sociales.

ra sus derechos y obligaciones. Recién en 1923 se firma un decreto que establecía las condiciones para el ingreso al Servicio Exterior y en 1947 se sanciona la ley 12.951 que unifica las carreras Diplomática y Consular y establece que el ingreso se efectuaría por el cargo inferior del escalafón. ver: Rizzo,(2012) y De Marco,(2013)

${ }^{14}$ José León Suarez(1872-1927), profesor de las materias de Derecho Internacional y Derecho Diplomático. Contribuyó a la creación del Ministerio de Agricultura en 1897 y fue fundador de la Facultad de Ciencias Económicas de la UBA en 1913 y Decano de la misma entre 1921 y 1924.

${ }^{15}$ La Escuela de Diplomacia dejó de funcionar en 1929, luego de la muerte de su fundador y la carrera se eliminó de los planes de estudios. De Marco,(2013).

${ }^{16}$ Enrique Ruíz Guiñazú(1884-1967). Abogado por la Facultad de Derecho y Ciencias Sociales de la UBA, docente de esa institución entre 1912 y 1930 dictando la materia Finanzas y Economía Politica, en la carrera de Diplomacia. Secretario de Hacienda en la ciudad de Buenos Aires (1908-1914) y a partir de 1930 se dedicó a la carrera diplomática. ${ }^{17}$ BMSA, (1912):27. 


\section{El MSA y el ambiente internacional}

Ahora bien, en tanto el MSA se presentaba como una institución orientada al estudio práctico de los problemas sociales que tenía el objetivo de colaborar en la obra del progreso nacional, el ambiente internacional se tornaba un factor clave ya que ofrecía un campo para lo observación de «cuestiones que residentes en el exterior no son por eso ajenas al interés del propio país, pudiendo influir en las relaciones políticas y económicas, en los tratados de comercio y en el régimen aduanero y en las convenciones internacionales». ${ }^{18}$

Estas concepciones llevaron a que el MSA se vincule con oficinas públicas en distintos países e instituciones internacionalistas, ${ }^{19}$ con el fin de realizar estudios e intercambiar informaciones. Para lo cual se abocaron a la constitución de una red de contactos con ministros plenipotenciarios, cónsules generales y cónsules que fueron nombrados como delegados del MSA en el extranjero y que garantizaron la presencia de la institución en diversos países y eventos, facilitando el acceso a documentos e informes sobre temas sociales. En esa línea invitaron a personalidades extranjeras a dar charlas y conferencias, se presentaron a congresos y exposiciones en representación propia y de la Argentina, y organizaron viajes de estudios. ${ }^{20}$ La consolidación de las redes fue crucial para el desarrollo del MSA ya que contribuyó a reforzar su identidad a nivel local e internacional como un instituto de estudios sociales y le permitió presentarse como una Institución federadora.

No obstante, el estallido del conflicto bélico tuvo fuertes resonancias en el desarrollo Institucional ya que le impidió continuar con el programa de acción que se había planteado originalmente. De esto es ilustrativa la carta enviada por el agente del MSA con asiento en Berlín, Gustavo

\footnotetext{
${ }^{18}$ Amadeo,(1910):18

${ }^{19}$ Entre ellas los Museos Sociales de Paris, Barcelona y Budapest, al Museo Obrero de Múnich, al Museo de Higiene de Dresde, al Museo de Charlotenburgo, la Oficina de Seguro Obrero del Imperio Alemán, los Servicios Sociales de Washington y Londres, el Instituto Internacional de Agricultura de Roma, la Oficina de Repúblicas Americanas, la Sociedad Humanitaria de Milán y el Instituto de Reformas Sociales de Madrid.

${ }^{20}$ Crean las bibliotecas argentinas en Berlín, Bruselas, en el Museo Internacional y en la biblioteca de T. Roosevelt; invitan a L.Mabilleau(1912) y T.Rooselvet(1913), organizan presentaciones en exposiciones internacionales: Gante(1913), Génova(1914) y firman Convenios de Reciprocidad con el Museo Social de París(1912) y con la Unión de Asociaciones Internacionales(1913).
} 
Niederlein, donde comunicaba que «la horrorosa guerra europea ha paralizado ahora del todo la obra de mi Agencia del Museo Social Argentino en Europa». ${ }^{21}$

\section{Solidaridad continental}

El nuevo contexto internacional ponía en crisis los objetivos de cooperación, solidaridad y propaganda propios de la institución, obligando a la dirección a reconfigurar su posición y reorientar las estrategias de acción. En ese marco, los sucesos vinculados a la inseguridad del trasporte marítimo operaron como oportunidad.

De esta manera, la Gran Guerra se constituyó en un problema de abordaje central para el MSA y el Boletín operó como una caja de resonancia, recogiendo alguno de los debates nacionales e internacionales. Así, a fines del 1914 aparece el primer artículo vinculado al conflicto, ${ }^{22}$ que fue seguido por la reproducción de una selección del capítulo Sofismas a favor de la Guerra ${ }^{23}$ del libro Cours d'enseignement pacifiste de A. Séve, publicado en Paris en 1910 y por una serie de artículos firmados por Bott ${ }^{24}$ y Otlet, ${ }^{25}$ promotores de la Unión de Asociaciones Internacionales, de la que participaba el MSA. En esos artículos se observa una reflexión vinculada a la importancia de la paz y el papel de los países neutrales frente al conflicto bélico y al rol de las instituciones sociales como potencias intelectuales capaces de promover la solidaridad en el continente, que le darán un sustento a las acciones que impulsará la institución estos años.

Si bien la Argentina, simultáneamente con Estados Unidos y el resto de los países de América Latina, había declarado tempranamente la neutralidad, ${ }^{26}$ a medida que el conflicto avanzaba la región comienza a

\footnotetext{
${ }^{21}$ MSA, (1914):478

${ }^{22}$ El momento económico, por Déccheri, BMSA, (1914):433

${ }^{23}$ La Guerra, BMSA, (1914), N³5/36: 525-544

${ }^{24}$ América ante la catástrofe, BMSA,(1915), $\mathrm{N}^{\circ} 37 / 38 ;$ La orientación del nacionalismo argentino, BMSA,(1915), $\mathrm{N}^{\circ} 39 / 40$; La politica del A, B, C, BMSA,(1915), $\mathrm{N}^{\circ} 43 / 44$; El Aislamiento Pacifico de América, BMSA,(1915), $\mathrm{N}^{\circ} 45 / 46$; El pacifismo luego de la gran guerra, BMSA(1916), $\mathrm{N}^{\circ} 49 / 50-51 / 52$.

${ }^{25}$ La supresión de la guerra por la confederación mundial de los estados, BMSA (1915), N³9/ 40; La politica Internacional Americana, BMSA,(1915), N 45/46.

${ }^{26}$ La neutralidad americana estuvo facilitada en principio por la lejanía del conflicto, en ese sentido la guerra era vivida como una experiencia ajena; asimismo, dada la vinculación de
} 
verse involucrada en el conflicto, ya que, por un lado, desde los primeros meses de la conflagración, la guerra naval llevó el enfrentamiento a aguas americanas ${ }^{27}$ y, por el otro, la eliminación por parte de Gran Bretaña de la diferencia entre contrabando absoluto y relativo, ocasionó que pasen a tener «el mismo tratamiento las municiones de guerra que los artículos de primera necesidad». ${ }^{28}$ En este contexto, la dirigencia del MSA se mostró alarmada por:

«la forma en que algunas de las naciones beligerantes interpretaban las reglas del derecho internacional referentes al contrabando de guerra y a consecuencia de lo cual proceden al apresamiento de buques mercantes de banderas neutrales, procedentes de los puertos americanos del atlántico y del pacifico, o de sus cargamentos» ${ }^{29}$

La Dirección evaluaba que si el comercio marítimo no se regularizaba «mediante alguna garantía nacional o internacional» la situación financiera argentina podría sufrir quebrantos irreparables, echando por la borda el optimismo inicial con el que fue analizada la situación económica que la guerra podría generar para la Argentina. ${ }^{30}$ La preocupación radicaba en que, si bien el Presidente Victorino de la Plaza había declarado la neutralidad, el Senado no había ratificado la subscripción a la Convención de la Haya que fijaba derechos y obligaciones de los neutrales, ${ }^{31}$ poniendo al país en una situación vulnerable. ${ }^{32}$

La guerra generó un notable interés en la sociedad Argentina, que incluyó, en los primeros años, manifestaciones pacifistas, actividades de caridad, movilizaciones de colectividades de inmigrantes y la organización de comités patrióticos. ${ }^{33}$ En ese contexto de intenso activismo social vin-

los países americanos a capitales europeos, la neutralidad evitaba poner en riesgo las relaciones económicas y, por otro lado, pueden mencionarse factores de orden cultural y social, vinculados al alto componente migratorio de los países latinoamericanos. Sobre la neutralidad americana ver: Compagnon,(2009) y Palamara,(2015).

${ }^{27}$ Rinke y Kriegesmann, (2015).

${ }^{28}$ Govi, (2013): 9

${ }^{29}$ BMSA, (1914):497

${ }^{30}$ El momento económico, por Déccheri, BMSA, (1914), N³4

${ }^{31}$ La Argentina suscribió a la Convención en 1907

${ }^{32}$ Por decreto de 5-8-1914 Victorino de la Plaza declara la neutralidad y entre el 05/08/ 1914 al 31/08/1916 se emiten 7 decretos de neutralidad, ver: Lascano,(1974) y Lanus, (2001).

${ }^{33}$ ver: Tato, (2007) y (2010). 
culado con la guerra, la dirección del MSA, basándose en su «autoridad moral» y en «las circunstancias de haber promovido, con éxito notorio, otras formas de solidaridad continental», ${ }^{34}$ solicita al Gobierno Nacional, por medio de una nota con fecha 4 de noviembre de 1914 dirigida al Ministro de Relaciones Exteriores y Culto, Sr. José Luis Murature, que inicie un acuerdo panamericano, a favor del comercio neutral. ${ }^{35}$ América pacífica «debe sostenerse a sí misma, apoyada en el derecho y sostenida por la fuerza formidable de la solidaridad continental» afirmaba Emilio Frers, Presidente del MSA.

La propuesta del MSA estaba en sintonía con las acciones iniciadas por el gobierno nacional por intermedio del embajador argentino en Washington, Dr. Rómulo Naon, ante el Secretario de Estado de los Estados Unidos, con el objetivo de tratar los derechos y obligaciones de los países neutrales y promover un acuerdo panamericano ante la Junta Directiva de la Unión Panamericana. ${ }^{36}$ En ese marco, el Ministro, si bien alienta al MSA para que difunda sus ideas, no le otorga un apoyo formal. El Presidente, Dr. Frers, visiblemente contrariado, manifiesta que «la actuación del $\mathrm{Mu}-$ seo no debía terminar en este asunto con la propaganda aconsejada por el Ministro de relaciones exteriores». ${ }^{37}$ De esta manera, el Consejo Superior decide que el MSA debe asumir un rol en la promoción de la defensa del derecho de los neutrales y prepara una circular dirigida a todas las instituciones universitarias y económicas de América Latina, con el fin de consultarles respecto a la utilidad y conveniencia de:

«gestionar ante los gobiernos americanos la adopción de un principio internacional que establezca la neutralidad absoluta del comercio mercante entre los pueblos del continente, siempre que dicho comercio se realice de acuerdo con ciertas reglas que deberán convenirse por los gobiernos» ${ }^{38}$

\footnotetext{
${ }^{34}$ El Museo Social Argentino invitó al ex Presidente de los Estados Unidos Theodore Roosevelt a dar una serie de charlas a la Argentina. De esta visita surge la posibilidad de colaborar institucionalmente con la División Internacional de intercambio y Educación de la Dotación Carnegie por la Paz Universal en lo referente al canje de información y de experiencias. BMSA, (1914):372-376. En relación con la invitación a Roosevelt, puede consultarse el sitio digital dedicado al presidente (http://www.theodorerooseveltcenter.org/ ), donde figura el discurso que éste dio en el MSA y hay fotos del encuentro y otras cuestiones relativas a su visita a la Argentina.

${ }^{35}$ BMSA, (1914):409

${ }^{36}$ Escudé y Cisneros, (1999)

${ }^{37}$ En la sesión $\mathrm{N}^{\circ} 37$ del C.S

${ }^{38}$ BMSA, (1914):598
} 
y de presentarles una nueva fórmula de derecho internacional el Cabotaje Interamericano, que constaba de los siguientes principios:

1- El comercio marítimo de los países americanos entre sí, será considerado como cabotaje inter-americano siempre que se efectúe directamente entre puertos de países americanos no beligerantes y que las embarcaciones no se aparten de las rutas que se determinen, dentro de las aguas continentales de América

2- Las embarcaciones mercante que se encuentren ocupadas con el cabotaje interamericano deberán ser consideradas como neutrales, aunque naveguen bajo bandera de países que se encuentran en estado de guerra ${ }^{39}$

La figura de Cabotaje Interamericano elaborada por el MSA aludía a «(...) la idea de una hermandad más estrecha entre las naciones americanas, como si sus costas marítimas pertenecieran a una sola gran comunidad internacional deseosa de conservar la paz en medio de los desastres de la guerra Europa». ${ }^{40}$ El propósito del MSA con estas acciones era conseguir la adhesión de los gobiernos, de la prensa, de las instituciones científicas, sociales y comerciales, para promover un acuerdo entre los países de neutrales de América que permita disminuir los perjuicios directos que guerra les pudiese ocasionar: «lo que deseamos es que los pueblos americanos se vinculen y se aúnen en la defensa de sus intereses comunes y de sus derechos neutrales, a fin de dar a su gestión mayor autoridad moral y eficacia». ${ }^{41}$

De esta manera, la propuesta de la institución que se interrogaba sobre el rol del continente americano ante la guerra europea se articulaba al principio de la solidaridad continental. En esa línea, la dirección del MSA estimaban indispensable construir una nueva forma de ideal panamericano: «así los pueblos de Sud América comenzarán a convencerse que la unión de Americana es un ideal común a todos ellos y su apostolado no es una misión exclusivamente yankee». ${ }^{42}$ En efecto, para Ernesto J.J. de Bott, quien en estos años tuvo a su cargo la conceptualización de la posición del Instituto en relación a la política exterior, el Panamericanismo en

\footnotetext{
${ }^{39}$ BMSA, (1914):500-506.

${ }^{40}$ Museo Social Argentino, (1916):26-27

${ }^{41}$ Museo Social Argentino, (1916): 14

${ }^{42}$ BMSA, (1915):207. Declaraciones hechas por el C.S del MSA, con motivo de la firma de un convenio de reciprocidad con la Dotación de Carnegie, en febrero de 1914.
} 
lugar de ser un movimiento liderado por los Estados Unidos, debía realizarse en la forma de un movimiento de cohesión de los pueblos americanos entre sí. Línea en la que puede ubicarse la importancia que el teórico le otorgaba a la actuación que Argentina, Brasil y Chile ${ }^{43}$ desempeñaron como mediadores ante el conflicto de Estados Unidos y México en $1914,{ }^{44}$ ya que según su perspectiva apuntaba a construir una política tendiente a neutralizar el carácter tutelar de la doctrina Monroe ${ }^{45}$. Bott estimaba que el arbitraje del $\mathrm{ABC}$ había sido un buen antecedente para propiciar un Panamericanismo con posibilidades de lograr la unificación de la potente intelectualidad americana. En esa orientación, sus escritos vislumbraban la imagen de una América pacificadora frente a la Europa en guerra:

Por primera vez se unen naciones independientes, sin constituir una amenaza para nadie. (...) Por primera vez parece deberse realizar con todas sus consecuencias la cooperación de los Estados y la democracia de las naciones. Surge una gran potencial para el bien, que no necesita cimentarse sobre la violencia ni emplearse como arma. Es un núcleo, un primer núcleo formado por la condensación parcial de las fuerzas latentes que aparecen en toda américa, de norte a sud, como una esperanza suprema para la civilización ${ }^{46}$

Para los miembros del MSA los efectos de la guerra habilitaron un creciente espíritu común entre los países del continente, capaz de «robustecer las causas y políticas determinantes de la corriente de acercamiento panamericano». ${ }^{47}$ La propuesta del MSA, con excepción de los cuestionamientos del Consejo de la Facultad de Derecho y Ciencias Sociales, que recomendaba «no inventar nuevas fórmulas» y recuperar aquellas propias del derecho internacional, fue apoyada por un número considerables de organismos e instituciones. Recibieron la adhesión de 146 personas, instituciones oficiales y particulares, empresarios y de representantes diplomáticos y consulares de 15 países Americanos -Estados Unidos, Uruguay, Paraguay, Chile, Bolivia, Costa Rica, Perú, Guatemala, Ecuador, Cuba, Venezuela, Brasil, El Salvador, México, Nicaragua-. Además tuvieron el

\footnotetext{
${ }^{43}$ Conocida como Arbitraje del ABC, que toma su nombre de las iniciales de los países actuantes.

${ }^{44}$ La politica del $A B C$, por Ernesto J.J. Bott, BMSA, (1915), N43/44.

${ }^{45}$ BMSA, (1915):329.

${ }^{46}$ BMSA, (1915):356.

${ }^{47}$ BMSA, (1915):41.
} 
apoyo de legaciones Argentinas, ministros y cónsules argentinos en Europa y de ministros europeos en Argentina. Asimismo, diversos diarios de América publicaron notas para promocionar la campaña del MSA. ${ }^{48} \mathrm{La}$ totalidad de las respuestas y comunicaciones recibidas fueron transcriptas en las páginas del Boletín bajo el titulo El Museo Social Argentino y la neutralidad Americana (cabotaje interamericano). ${ }^{49}$ Ernesto Bott elaboró un estudio que se tituló El Aislamiento pacifico de América, ${ }^{50}$ publicado en el Boletín del MSA, en la Revista de Ciencias Económicas ${ }^{51}$ y en La Argentina Económica ${ }^{52}$, que fundamentaba conceptualmente la propuesta de la institución. En esa línea el discurso, El deber de América ante la presente crisis, pronunciado por el Dr. Leo S. Rowe ${ }^{53}$ y el texto, Politica Internacional Americana, de Paul Otlet ${ }^{54}$, aunque desde posiciones diferentes, apoyaban teóricamente la figura creada por el MSA.

En diciembre de 1915, el acorazado ingles Orama apresa, en las costas uruguayas, del Buque Presidente Mitre - perteneciente a la Línea Nacional del Sur, una firma subsidiaria de la Hamburg-Südamerikanische-Dampfschifffahrtgesellschaft (Compañía de Navegación a Vapor Hamburgo Sudamericana) - que formaba parte de la flota Mercante Argentina. Inglaterra, con el fin de obstruir el abastecimiento alemán, tenía buques de guerra patrullando las costas del litoral marítimo argentino y la boca del Rio de la Plata. ${ }^{55}$ Estos hechos generaron rechazo por parte de algunos sectores de la opinión pública, que organizaron manifestaciones contra los

${ }^{48}$ Entre ellos el Boston Daily Advertiser (Boston, EE.UU); el Diario del Plata (Montevideo, con notas el 15 y 21 de febrero de 1915); La Estrella del Panana (Panamá, con notas el 21 enero 1915); La Prensa (Lima, Perú, con notas del 28 de febrero 1915 y del 23 de marzo 1915); El comercio (Lima, Perú, con nota del 4 de marzo de 1915) La Época (Lima, Perú, con nota del 13 de marzo de 2015); El Triunfo (Habana, Cuba); Cuba Ilustrada (Habana, Cuba) y de la Revista Económica (Tegucigalpa, Honduras, con nota de septiembre de 1915).

${ }^{49}$ El Museo Social Argentino y la neutralidad Americana (cabotaje interamericano) BMSA (1914): $\mathrm{N}^{\circ} 35 / 36 ;$ BMSA, (1915): $\mathrm{N}^{\circ} 37 / 38-39 / 40-41 / 42-45 / 46$.

${ }^{50}$ El Aislamiento pacifico de América, BMSA, (1915): $\mathrm{N}^{\circ} 45 / 46$.

${ }^{51}$ Revista de Ciencias Económicas, publicación periódica del Centro de Estudiantes de Ciencias Económicas, (1915), marzo-abril y mayo.

${ }^{52}$ La Argentina Económica Año 1915 N 116 y 118.

${ }^{53}$ El deber de América ante la presente crisis, BMSA, (1915): $\mathrm{N}^{\circ} 41 / 42$.

${ }^{54}$ La Politica internacional americana, por Paul Otlet, BMSA, (1915): $\mathrm{N}^{\circ} 45 / 46$.

${ }^{55}$ En 1909 Londres había establecido que se declaraba el carácter neutral o enemigo de un buque por el pabellón que tenía derecho a llevar, sin embargo, en octubre de 2015 la nacionalidad de los buques comienzan a ser reconocidas por la propiedad de los mismos y no por su bandera. Lanus, (2015):68-69. 
ingleses. El apresamiento del Mitre y las negociaciones diplomáticas para su devolución colocaron a la dirección de la política exterior del gobierno de Victorino de la Plaza en el centro de las críticas y el diputado Estanislao Zeballos pide la renuncia del Canciller Murature. ${ }^{56}$

En ese marco, el MSA, relanza la campaña sobre el Cabotaje Interamericano ${ }^{57}$, dirigida, ahora, a los intelectuales e instituciones de América ya que, desde la perspectiva del Consejo Superior, era a ellos a quienes «les correspondía prestar una acogida práctica a la iniciativa del MSA en tanto los gobiernos no realicen algún acto decisivo en defensa de los intereses comunes». ${ }^{58}$ Por su parte, la reunión en abril de 1916 de la Alta Comisión Financiera Internacional ${ }^{59}$ y de la Alta Comisión Internacional de Legislación Uniforme en Buenos Aires le da un renovado impulso a las acciones, por lo que envían sendas Notas a la Comisión Nacional de Legislación Uniforme para que patrocine la inclusión en su programa de estudio de la figura de Cabotaje Interamericano ${ }^{60}$ y publican el libro El aislamiento pacifico de América. Documentación relativa a la campaña del Museo Social Argentino por la neutralización del tráfico maritimo internacional, que compilaba todas las comunicaciones sobre el tema.

Ahora bien, la propuesta del MSA intenta, en un contexto de amplio activismo social vinculado con el conflicto bélico, promover la organización de la Solidaridad Americana para la defensa de los intereses de la región. Desde esa perspectiva, la neutralidad Americana operó como una oportunidad para presentar una alternativa al Panamericanismo impulsado por el Estados Unidos. No obstante, a medida que el conflicto avanzaba se dificultaba sostener una posición americana común. Luego del ingreso de Estados Unidos en la guerra solo mantendrán la neutralidad Argentina, Chile, Colombia, México, Paraguay, El Salvador y Venezuela. ${ }^{61}$ Por su parte, en el medio local, comienzan a tomar forma grupos de opinión y de presión organizados tras los debates neutralistas y rupturistas,

\footnotetext{
${ }^{56}$ Escudé y Cisneros,(1999) y Lascano,(1974).

${ }^{57}$ Las comunicaciones de la nueva campaña fueron publicadas en el Boletín bajo el nombre de Cabotaje interamericano (el aislamiento pacifico de América) BMSA,(1916), №49/50$51 / 52-53 / 54$ y $55 / 56$

${ }^{58}$ Museo Social Argentino, (1916):3

${ }^{59}$ Creada en el Primer Congreso Financiero Panamericano, reunido en Washington en 1915, para facilitar la ejecución de las resoluciones,

${ }^{60}$ Sesión $\mathrm{N}^{\circ} 49$ del C.S del 7-12-1915

${ }^{61}$ Luego del 1917 solo Argentina, Chile, Colombia, México, Paraguay, El Salvador y Venezuela mantienen la neutralidad. Palamara,(2015) y Compagnon,(2009).
} 
que dan cuenta de profundas fracturas sociales. ${ }^{62} \mathrm{~A}$ esto se le sumaba el intento, infructuoso, del Yrigoyenismo ${ }^{63}$ de organizar una estrategia común de los países latinoamericanos a través de una Conferencia de Neutrales. De esta manera, en este nuevo escenario, donde ya no eran posibles las prédicas de conciliación, el Museo Social Argentino abandona la propuesta del cabotaje interamericano.

\section{Conclusiones}

Con este artículo nos propusimos iluminar las actuaciones de una institución privada que intentó, en el marco de la primera guerra, asumir un papel en defensa de los intereses nacionales promoviendo la solidaridad continental. Si bien la Guerra tuvo un fuerte impacto en las actividades que estaba realizando el MSA, la dirección de la institución logra reconfigurar las estrategias y orientar sus acciones a América.

El llamamiento a los pueblos de América a unirse en la solidaridad continental, promovido por el MSA, apuntaba a establecer otros vínculos en el continente, en los que la Argentina y la propia institución tendrían un lugar privilegiado. Así, el MSA, que pretendía colaborar en la obra del progreso nacional, logró desplegar una serie de estrategias en defensa de los derechos de los países neutrales, iniciando una intensa campaña con la intención de presionar a la opinión pública y a los Estados latinoamericanos para lograr que sea sancionada una nueva figura de derecho internacional: el cabotaje interamericano. Esto llevó a la institución, por momentos, a actuar en paralelo a las acciones que estaba llevando adelante el Estado nacional. Las gestiones realizadas colaboraron en la profundización de las relaciones con las instituciones americanas. En ese sentido, la guerra fue tomada como una instancia de recomposición de la identidad continental, una nueva forma de ideal panamericano.

Por último, sin bien el hundimiento de varias naves de bandera argentina por parte del Imperio Alemán a partir de abril de $1917^{64}$ ubicó la política exterior argentina en el centro de las discusiones públicas ${ }^{65}$ el MSA no aborda el tema. En ese sentido, por un lado, el ingreso de los Estado

\footnotetext{
${ }^{62}$ Ver: Tato,(2008) y Cagni,(2014).

${ }^{63}$ Hipólito Yrigoyen asume la presidencia el 12 de octubre de 1916.

${ }^{64} \mathrm{El} 4$ de abril hundimiento del vapor Monte Protegido y de las naves Oriana y Toro, 6 de junio y el 22 de junio respectivamente

${ }^{65}$ ver: Tato, (2007), (2008) y (2010)
} 
Unidos en la guerra y la ruptura de la neutralidad americana y, por el otro, la agudización de los conflictos internos, ocasionan que el nuevo Consejo Superior -que entra en funciones a principios de 1917- ante la imposibilidad de promover el proyecto de solidaridad continental, oriente los estudios del MSA hacia otros temas. Así, los artículos referidos a la neutralidad y el cabotaje interamericano dejan de aparecer en el Boletín institucional.

\section{Bibliografía}

AMADEO, Tomas, (1910), Museo Social de Buenos Aires. Fundamentos y Anteproyecto, Buenos Aires: Coni.

AMADEO, Tomas, (1939), «La enseñanza de la agricultura en la escuela primaria» en El Falso dilema Fascismo o Bolcheviquismo, Buenos Aires: Librería del Colegio, pp.219-248.

CAGNI, Horacio, (2014), «La neutralidad Argentina ante el impacto cultural de la Gran Guerra», en PolHis. Revista Bibiográfica del Programa Interuniversitario de Historia Política, año 7, $\mathrm{N}^{\circ} 14$

COMPAGNON, Oliver, (2009), «Entrer en guerre? Neutralité et engagement de l'Amérique latine entre 1914 et 1918», en Relations internationales, $\mathrm{N}^{\circ} 137$, pp. 31-43, en línea: www.cairn.info/revue-relations-internationales-2009-1-page-31.htm.

COMPAGNON, Oliver, (2014), América Latina y la Gran Guerra. El adiós a Europa (Argentina y Brasil, 1914-1939), Buenos Aires: Crítica

DE MARCO, Miguel Ángel, (2013), «La formación de expertos y diplomáticos en la Facultad de Ciencias Económicas, Comerciales y Políticas de Rosario, 1920-1968» en Cuadernos de Politica Exterior Argentina, $\mathrm{N}^{\mathrm{O}} 112$ (abril-junio), Rosario: CERIR, pp.1-32

ESCUDÉ, Carlos y CISNEROS, Alejandro (1999) «Las relaciones exteriores de la argentina consolidada (1881-1942)» en Historia general de las relaciones exteriores de la República Argentina, tomo VIII, GEL, en línea: http://www.argentina-rree.com/home_nueva.htm

GIRBAL DE BLACHA, Noemí y OSPITAL, María Silvia, (1986), «Elite, cuestión social y apertura política en la Argentina (1910-1930): La propuesta del Museo Social Argentino» en Revista de Indias, XLVI $\mathrm{N}^{\circ} 178$, pp.609-625.

GOVI, Pablo, (2013), Estudio comparativo de la politica exterior argentina durante los gobiernos de Victorino de la Plaza e Hipólito Yrigoyen en el 
contexto de la Primera Guerra Mundial, Tesis de Maestría, Universidad de Belgrano, en línea, http://www.ub.edu.ar/investigaciones/ tesis/82_Govi.pdf

HORNE, Janet (1998) «Le libéralisme à l'épreuve de l'industrialisation: la réponse du Musée social», en Le Musée social en son temps, Colette, CHAMBELLAND (Ed), Paris: Presses de L'Ecole Normale Supérieure, pp.13-25

LASCANO, Alen, (1974), Yrigoyen y la Gran Guerra, Buenos Aires: Korrigan.

LANUS, Juan Archivaldo, (2001), Aquel Apogeo, politica internacional Argentina 1910-1939, Buenos Aires: Emece.

NOVICK, Alicia, (1993), «El Museo Social Argentino. La ciudad desde el Campo» en Seminario de Critica-IAA, №46, en línea: http:// www.iaa.fadu.uba.ar/publicaciones/critica/0046.pdf

PALAMARA, Graziano, (2015), «Entre guerra y paz. América Latina frente a la tragedia del primer conflicto mundial», en Anuario Colombiano de Historia Social y de la Cultura, N42.2, pp.103-126.

PELOSI, Hebe Carmen, (2000), El Museo Social Argentino y La Universidad del Museo Social Argentino. Historia y Proyección (1911-1978), Buenos Aires: UMSA

RAMÍREZ BACCA, Renzo, (2015), «Estudios sobre la Primera Guerra Mundial en América Latina. Una mirada comparada» en Anuario Colombiano de Historia Social y de la Cultura, 42, 2, pp.43-73, en línea: http://dx.doi.org/10.15446/achsc.v42n2.53329.

RINKE, Stefan, (2014), «Historiography 1918-Today (Latin America)» en 1914-1918-online. International Encyclopedia of the First World War; Berlin: Freie Universität Berlin; DOI: http://dx.doi.org/10.15463/ iel418.10501RINKE, Stefan y KRIEGESMANN, Karina (2015), «Latin America», en 1914-1918-online. International Encyclopedia of the First World War, Berlín: Freie Universität Berlin, 2015-11- 05, DOI: http://dx.doi.org/10.15463/ie1418.10760.

RIZZO, Natalia, (2012), «Los profesionales de Estado en la Argentina: el caso del Instituto Superior de la Nación (ISEN)» en Cuadernos de Politica Exterior Argentina, $\mathrm{N}^{\circ}$ 107, enero-marzo, Rosario: CERIR, pp.1-22

SURIANO, Juan, (2000), La cuestión social en Argentina 1870-1943, Buenos Aires: La Colmena 
SURIANO, Juan, (2013), «El mundo como un taller de observación. La creación del Departamento Nacional del trabajo y las influencias internacionales», en Circulación internacional de saberes y prácticas institucionales en la consolidación del Estado Social en Argentina. Siglos XIX y XX, GONZÁLEZ LEANDRI y GONZÁLEZ BERNALDO DE QUIRÓS (coord.), Revista de Indias, 73, 257, pp.107130. http://revistadeindias.revistas.csic.es/index.php/revistadeindias/ article/view/919/992

TATO, María Inés, (2007), «Ciudadanos en movimiento: la sociedad porteña y la Primera Guerra Mundial», en II Jornadas sobre la Politica en Buenos Aires en el Siglo XX, Lugar: Tandil.

TATO, María Inés, (2008), «La disputa por la argentinidad. Rupturistas y neutralistas durante la Primera Guerra Mundial», en Temas de Historia Argentina y Americana, $\mathrm{N}^{\circ} 13$, pp. 227 - 250

TATO, María Inés, (2010), «En el nombre de la Patria: asociacionismo y nacionalismo en la Argentina en torno de la Primera Guerra Mundial» Rey Tristán, Eduardo; Calvo González, Patricia. XIV Encuentro de Latinoamericanistas Españoles: Congreso Internacional, Sep.2010, pp.303-315, 2010, Cursos e Congresos; 196. < halshs00529306>

TATO, María Inés, (2014), «La Gran Guerra en la historiografía argentina. Balance y perspectivas de investigación» en Iberoamerican, XIV, 53, Madrid: Iberoamericana/Vervuert, pp.91-101

TOPALOV, Christian, (1990), «De la «cuestión social» a los «problemas urbanos»: los reformadores y la población de las metrópolis a principios del siglo XX» en Revista Internacional de Ciencias Sociales, $\mathrm{N}^{\circ}$ 125.

ZIMMERMANN, Eduardo, (1995), Los liberales reformistas. La cuestión social en la Argentina. Buenos Aires: Sudamericana

ZIMMERMANN, Eduardo, (2013), «»Un espíritu nuevo»: la cuestión social y el Derecho en la Argentina (1890-1930)» en Circulación internacional de saberes y prácticas institucionales en la consolidación del Estado Social en Argentina. Siglos XIX y XX, GONZÁLEZ LEANDRI, GONZÁLEZ BERNALDO DE QUIRÓS (coord.), Revista de Indias, 73, 257, pp.81-106. http://revistadeindias.revistas.csic.es/ index.php/revistadeindias/article/viewArticle/918 